\title{
Topical Delivery of Muscarinic Receptor Antagonists Prevents and Reverses Peripheral Neuropathy in Female Diabetic Mice
}

\author{
(D) Corinne G. Jolivalt, Katie E. Frizzi, May Madi Han, Andre J. Mota, Lucie S. Guernsey, \\ (D)Lakshmi P. Kotra, (1) Paul Fernyhough, and (1) Nigel A. Calcutt \\ Department of Pathology, University of California San Diego, San Diego, California (C.G.J., K.E.F., M.M.H., A.J.M., L.S.G., \\ N.A.C.); Winsantor Inc. (K.E.F). Center for Molecular Design and Preformulations and Krembil Research Institute, University \\ Health Network and Leslie Dan Faculty of Pharmacy, University of Toronto, Toronto, Ontario, Canada (L.P.K.); and Department of \\ Pharmacology and Therapeutics and Division of Neurodegenerative Disorders, St. Boniface Hospital Albrechtsen Research \\ Center, University of Manitoba, Winnipeg, Manitoba, Canada (P.F.)
}

Received January 29, 2020; accepted April 20, 2020

\begin{abstract}
Muscarinic antagonists promote sensory neurite outgrowth in vitro and prevent and/or reverse multiple indices of peripheral neuropathy in rodent models of diabetes, chemotherapyinduced peripheral neuropathy, and HIV protein-induced neuropathy when delivered systemically. We measured plasma concentrations of the $M_{1}$ receptor-selective muscarinic antagonist pirenzepine when delivered by subcutaneous injection, oral gavage, or topical application to the skin and investigated efficacy of topically delivered pirenzepine against indices of peripheral neuropathy in diabetic mice. Topical application of $2 \%$ pirenzepine to the paw resulted in plasma concentrations 6 hours postdelivery that approximated those previously shown to promote neurite outgrowth in vitro. Topical delivery of pirenzepine to the paw of mice with streptozotocin-induced diabetes dose-dependently $(0.1 \%-10.0 \%)$ prevented tactile allodynia, thermal hypoalgesia, and loss of epidermal nerve fibers in the treated paw and attenuated large fiber motor nerve
\end{abstract}

conduction slowing in the ipsilateral limb. Efficacy against some indices of neuropathy was also noted in the contralateral limb, indicating systemic effects following local treatment. Topical pirenzepine also reversed established paw heat hypoalgesia, whereas withdrawal of treatment resulted in a gradual decline in efficacy over 2-4 weeks. Efficacy of topical pirenzepine was muted when treatment was reduced from 5 to 3 or 1 day/wk. Similar local effects were noted with the nonselective muscarinic receptor antagonist atropine when applied either to the paw or to the eye. Topical delivery of muscarinic antagonists may serve as a practical therapeutic approach to treating diabetic and other peripheral neuropathies.

\section{SIGNIFICANCE STATEMENT}

Muscarinic antagonist pirenzepine alleviates diabetic peripheral neuropathy when applied topically in mice.

\section{Introduction}

Muscarinic acetylcholine receptor antagonists have been in clinical use for decades to treat such diverse conditions as Parkinson disease, peptic ulcers, overactive bladder, chronic obstructive pulmonary disease, asthma, and motion sickness, with specific drug use depending on muscarinic receptor subtype selectivity and tissue accessibility (Kruse et al., 2014). Despite the widely studied and pharmacologically manipulated role of muscarinic receptors in modulating synaptic function in the central (Erskine et al., 2019; Moran et al., 2019) and autonomic (Giglio and Tobin, 2009; Tobin et al., 2009) nervous systems, it has only recently become

This work was supported by National Institutes of Health, National Institute of Neurologic Disorders and Stroke [Grant RO1-NS081082 to N.A.C.].

P.F. and L.P.K. are cofounders, shareholders, and serve on the Board of Directors of Winsantor Inc. N.A.C. is a cofounder and shareholder in Winsantor Inc. K.E.F. is an employee of Winsantor Inc. and UCSD.

https://doi.org/10.1124/jpet.120.265447. apparent that peripheral sensory neurons maintained in vitro after extraction from adult rodents experience tonic cholinergic suppression of maximal mitochondrial respiratory capacity and neurite outgrowth (Calcutt et al., 2017). Inhibition of the $M_{1}$ subtype of the muscarinic receptor $\left(M_{1} R\right)$, which is expressed by adult sensory neurons, is critical to these effects as neurite outgrowth from sensory neuron cultures derived from adult rodents is increased by $\mathrm{M}_{1} \mathrm{R}$ knockout and by diverse $\mathrm{M}_{1} \mathrm{R}$-specific and -selective antagonists. In contrast, muscarinic receptor antagonists selective for the $\mathrm{M}_{2-4} \mathrm{R}$ subtypes do not promote neurite outgrowth, whereas the muscarinic receptor agonist muscarine suppressed neurite outgrowth (Calcutt et al., 2017).

The therapeutic implications of cholinergic constraint of sensory neurons include the potential for muscarinic antagonists to promote nerve regeneration after injury and to enhance mitochondrial activity in neurons under metabolic stress. It is becoming increasingly apparent that a number of peripheral neuropathies, including those associated with

ABBREVIATIONS: AMPK, AMP-activated protein kinase; $\mathrm{AUC}_{0-\mathrm{t}}$, Area Under the Curve from 0 to $\mathrm{t}$; IENF, intraepidermal nerve fiber; MNCV, motor nerve conduction velocity; MR, muscarinic receptor; STZ, streptozotocin; $T_{\max }$, Time of first occurence of $\mathrm{C}_{\max }$. 
diabetes (Fernyhough, 2015; Chandrasekaran et al., 2019), chemotherapy (Trecarichi and Flatters, 2019), and HIV infection (Roda and Hoke, 2019), share a common pathogenesis centered on impaired mitochondrial activity. In a recent study, mice lacking the $\mathrm{M}_{1} \mathrm{R}$ were protected from diabetesinduced neuropathy, whereas subcutaneous injection of selective $M_{1} R$ antagonists prevented and reversed multiple functional and structural indices of peripheral neuropathy in rodent models of diabetes, chemotherapy-induced neuropathy, and neuropathy caused by HIV-associated proteins (Calcutt et al., 2017). Efficacy of the $\mathrm{M}_{1} \mathrm{R}$-selective antagonists pirenzepine and VU0255035 was associated with activation of AMP-activated protein kinase (AMPK), augmentation of mitochondrial complex activity, and elevated expression of components of the respiratory electron transport chain (Calcutt et al., 2017). These studies highlight the therapeutic potential of $\mathrm{M}_{1} \mathrm{R}$-selective muscarinic antagonists against peripheral neuropathy.

A major concern regarding clinical use of antimuscarinic drugs is the potential for unwanted side effects due to the widespread distribution of muscarinic receptors and lack of receptor subtype specificity. Poor antibody specificity has hampered definitive distribution studies for $\mathrm{M}_{1} \mathrm{R}$ (Jositsch et al., 2009), but studies localizing $M_{1} R$ mRNA or identifying physiologic consequences of $M_{1} R$ knockout have offered clues. Other than being expressed by neurons and Schwann cells of the peripheral nervous system (Tata et al., 2000a,b; Loreti et al., 2006; Calcutt et al., 2017), $\mathrm{M}_{1} \mathrm{Rs}$ are also found in the alimentary tract and associated enteric nervous system and exocrine pancreas (Gautam et al., 2005; Tobin et al., 2009; Harrington et al., 2010), the cardiovascular system (Saternos et al., 2018), and epidermis (Ndoye et al., 1998; Kurzen et al., 2004). Moreover, the only known $M_{1} R$-specific antagonist is muscarinic toxin 7 (Krajewski et al., 2001; Servent et al., 2011), a peptide that is not currently a viable drug candidate. Pirenzepine may serve as an alternative candidate for manipulating the cholinergic constraint of peripheral sensory nerves in vivo, as it is $M_{1} R$-selective relative to the $M_{2-5} R$ subtypes (Eglen et al., 2001) and does not readily cross the blood-brain barrier, reducing the potential for disruption of central nervous system function compared with other muscarinic antagonists (Jaup and Blomstrand, 1980; Sethy and Francis, 1990). Pirenzepine was originally developed as an orally delivered drug to treat ulcers, acting locally in the stomach to reduce gastric acid secretion while having weak systemic side effects (Carmine and Brogden, 1985). Other approaches to reducing systemic side effects of antimuscarinics have included use of topical delivery (Sand, 2009). In the present study, we have extended studies of the therapeutic potential of muscarinic antagonists against diabetic neuropathy to address the viability of delivery by topical application to the skin or eye to treat multiple indices of peripheral neuropathy in a mouse model of type 1 diabetes.

\section{Materials and Methods}

Animals. All procedures on live animals were approved by the local Institutional Animal Care and Use Committee. Studies were performed in adult female C57 Bl/6J (\#000664; Jackson Laboratories) or female Swiss Webster (\#024; Charles River) mice maintained three to five per cage on TEK-Fresh bedding (\#7099; Envigo) under a 12-hour light/dark cycle with free access to water and food (5001 diet; Purina).
Only female mice were used to keep group variability to a minimum. We have previously reported that muscarinic antagonists are effective against indices of neuropathy in both male and female diabetic rodents (Calcutt et al., 2017). Insulin-deficient diabetes was induced by intraperitoneal injection of 90 (C57 Bl/6J mice) or $100 \mathrm{mg} / \mathrm{kg}$ (Swiss Webster mice) streptozotocin (STZ; Sigma) in sterile $0.9 \%$ saline on two consecutive days, with each injection following a 12-hour fast. Peripheral neuropathy in this model is not the result of direct STZinduced neurotoxicity (Davidson et al., 2009). Onset of hyperglycemia was confirmed 4 and 5 days after the second STZ injection and also at study end in blood obtained by tail prick from restrained but conscious animals using a strip-operated glucose meter (OneTouch UltraMini; Lifescan Inc.). Only mice with blood glucose concentrations above $270 \mathrm{mg} / \mathrm{dl}$ at both study onset and study end were included in data analysis. Deaths during the study period and animals that reverted to normoglycemia before study end are reported in Table 1.

Treatment. Pirenzepine dihydrochloride or atropine (Sigma) were prepared for topical application to the paw by mixing into a volume of sterile hydrogel (Intrasite \#66027313; Smith \& Nephew) immediately before application. Fifty microliters of pirenzepine/hydrogel was applied to the plantar surface of the hind paw of manually restrained conscious mice, and the paw was then enclosed in a plastic chamber that prevented the animal from ingesting or removing the drug preparation while allowing it to move freely within its cage. Vehicletreated animals received hydrogel alone. The chamber was left in place for $20 \mathrm{~min} /$ day, 5 days/wk, unless otherwise noted, after which it was removed, and the drug preparation was cleared with a tissue. For delivery to the eye, atropine was dissolved in ophthalmic gel (Genteal; Novartis), and $20 \mu \mathrm{l}$ was applied to the eye of a conscious, manually restrained mouse. The gel was left in place for 15 seconds before the animal was released. Vehicle-treated animals and/or the contralateral eye received ophthalmic gel alone. Plasma pirenzepine concentrations were measured by High Performance Liquid Chromatography-Mass Spectrometry with a lower detection limit of $2 \mathrm{nM}$ (Drumetrix Laboratories, Greensboro, NC) following delivery of $10 \mathrm{mg} / \mathrm{kg}$ pirenzepine by oral gavage or intraperitoneal injection to match doses previously effective against indices of neuropathy in diabetic rodents (Calcutt et al., 2017) or topical application of $2 \%$ gel based on unpublished preliminary data.

Electrophysiology. Conduction velocity of large myelinated motor fibers was measured in the sciatic nerve as described in detail elsewhere (Jolivalt et al., 2016). Briefly, mice were anesthetized with isoflurane, core and nerve temperatures were held at $37^{\circ} \mathrm{C}$ using heating pads and lamps, and fine needle stimulating electrodes were placed at the sciatic notch and Achilles tendon. Recording electrodes were inserted into the interosseus muscles of the ipsilateral paw. The sciatic nerve was stimulated using a PowerLab 4/30 to achieve maximal $\mathrm{M}$ wave amplitude $(0.2-1.0 \mathrm{mV}, 0.05$-millisecond square waves), and the resulting electromyogram was stored to a computer running LabChart Pro (AD Instruments). Motor nerve conduction velocity (MNCV) was calculated as the distance between stimulation sites divided by the latency of $\mathrm{M}$ wave peaks produced by stimulation at the two sites. The median of three separate measurements was used to represent MNCV for each animal.

Response to Pressure and Heat Stimuli. Hind limb withdrawal reaction to light touch and escalating heat stimuli were measured in conscious, unrestrained mice, as described in detail elsewhere (Jolivalt et al., 2016). Briefly, mice were allowed to acclimate to an observation chamber for 30 minutes prior to testing. Paw withdrawal to pressure applied at the plantar surface was measured using von Frey filaments applied sequentially in an updown protocol as originally described for rats (Chaplan et al., 1994) and subsequently modified for use in mice (Jolivalt et al., 2016). Paw response latency to surface heat escalating at a rate of $1^{\circ} \mathrm{C} / \mathrm{s}$ from a starting surface temperature of $30^{\circ} \mathrm{C}$ was measured using a Hargreaves apparatus. Triplicate measurements were made at 5 minute intervals, and the median was used to represent $50 \%$ paw withdrawal 
TABLE 1

Animal group data from all studies Data are group means \pm S.D.

Statistical comparisons by one-way ANOVA with Sidak's post hoc test.

\begin{tabular}{|c|c|c|c|c|c|}
\hline & $N$ & Study Deaths & Loss of Diabetes & Body Weight (g) & Plasma Glucose (mg/dl) \\
\hline \multicolumn{6}{|l|}{ Study 1 (Fig. 2) } \\
\hline Control + vehicle & 10 & 0 & 0 & $24.3 \pm 0.6$ & $159 \pm 12$ \\
\hline STZ + vehicle & 12 & 0 & 0 & $22.4 \pm 0.5^{* *}$ & $358 \pm 59 * * *$ \\
\hline $\mathrm{STZ}+0.2 \% \mathrm{PZ}$ & 12 & 0 & 1 & $21.1 \pm 1.3^{* *}$ & $386 \pm 111^{* * *}$ \\
\hline $\mathrm{STZ}+1.0 \% \mathrm{PZ}$ & 13 & 0 & 0 & $21.3 \pm 1.8^{* *}$ & $459 \pm 79 * * * \dagger$ \\
\hline $\mathrm{STZ}+2.0 \% \mathrm{PZ}$ & 9 & 0 & 3 & $21.5 \pm 1.3^{* *}$ & $483 \pm 98 * * * \dagger \dagger$ \\
\hline \multicolumn{6}{|l|}{ Study 2 (Fig. 3A) } \\
\hline Control + vehicle & 4 & $3^{a}$ & 0 & $22.8 \pm 0.7$ & $139 \pm 11$ \\
\hline Control + PZ & 7 & 0 & 0 & $22.8 \pm 0.4$ & $136 \pm 12$ \\
\hline STZ + PZ (withdrawal) & 9 & 3 & 0 & $20.8 \pm 0.8^{* * *}$ & $438 \pm 69 * * *$ \\
\hline $\mathrm{STZ}+\mathrm{PZ}($ reversal $)$ & 10 & 2 & 0 & $21.3 \pm 0.5^{* *}$ & $495 \pm 101 * * *$ \\
\hline \multicolumn{6}{|l|}{ Study 3 (Fig. 3B) } \\
\hline Control + vehicle & 10 & 0 & 0 & $27.6 \pm 2.1$ & $153 \pm 28$ \\
\hline STZ + vehicle & 10 & 0 & 0 & $23.4 \pm 2.2 * * *$ & $560 \pm 18 * * *$ \\
\hline $\mathrm{STZ}+\mathrm{PZ}(1 / \mathrm{wk})$ & 10 & 0 & 0 & $22.9 \pm 2.2^{* * *}$ & $587 \pm 28 * * *$ \\
\hline $\mathrm{STZ}+\mathrm{PZ}(3 / \mathrm{wk})$ & 7 & 3 & 0 & $23.3 \pm 2.8^{* *}$ & $593 \pm 19 * * *$ \\
\hline $\mathrm{STZ}+\mathrm{PZ}(5 / \mathrm{wk})$ & 11 & 0 & 0 & $22.5 \pm 1.4^{* * *}$ & $589 \pm 34^{* * *}$ \\
\hline \multicolumn{6}{|l|}{ Study 4 (Fig. 4) } \\
\hline Control + vehicle & 10 & 0 & 0 & $20.3 \pm 1.1$ & $131 \pm 15$ \\
\hline STZ + vehicle & 8 & 0 & 0 & $20.0 \pm 1.7$ & $437 \pm 92^{* * *}$ \\
\hline $\mathrm{STZ}+2.0 \% \mathrm{PZ}$ & 6 & 3 & 0 & $20.0 \pm 0.5$ & $434 \pm 132 * * *$ \\
\hline $\mathrm{STZ}+10.0 \% \mathrm{PZ}$ & 9 & 1 & 0 & $20.5 \pm 0.7$ & $415 \pm 149 * * *$ \\
\hline \multicolumn{6}{|l|}{ Study 5 (Fig. 5) } \\
\hline Control + vehicle & 10 & 0 & 0 & $28.9 \pm 1.3$ & $127 \pm 13$ \\
\hline STZ + vehicle & 9 & 1 & 0 & $26.9 \pm 1.8^{*}$ & $525 \pm 107^{* * *}$ \\
\hline $\mathrm{STZ}+2.0 \%$ atropine (paw) & 8 & 2 & 0 & $25.7 \pm 1.3 * * *$ & $542 \pm 100 * * *$ \\
\hline $\mathrm{STZ}+2.0 \%$ atropine (eye) & 9 & 0 & 1 & $27.1 \pm 1.3$ & $524 \pm 33 * * *$ \\
\hline
\end{tabular}

PZ, pirenzepine.

${ }^{*} P<0.05 ; * * P<0.01 ; * * * P<0.001$ vs. control + vehicle; ${ }^{\dagger} P<0.05 ;{ }^{\dagger \dagger} P<0.01$ vs. STZ + vehicle.

${ }^{a}$ Animals died during an equipment malfunction.

threshold (50\% paw withdrawal threshold in grams) and paw heat response latency (seconds).

Nerve Density in the Cornea and Paw. The density of sensory nerves in the corneal sub-basal nerve plexus of anesthetized and restrained mice was measured by corneal confocal microscopy, as described in detail elsewhere (Jolivalt et al., 2016). Five sequential images of the corneal sub-basal nerve plexus were obtained from isoflurane-anesthetized mice using a corneal confocal microscope (HRT3 with Rostock corneal module; Heidelberg Engineering Inc., Germany). Nerve occupancy was measured using an $8 \times 8$ grid overlaid on the images (Chen et al., 2013). Density of sensory nerve profiles in the epidermis of plantar hind paw skin was measured as described in detail elsewhere (Jolivalt et al., 2016). Briefly, plantar paw skin was removed at autopsy, immersion fixed in $4 \%$ paraformaldehyde, dehydrated in ethanol, and embedded in paraffin prior to cutting $6-\mu \mathrm{m}$ sections. Intraepidermal nerve fibers (IENF) were identified by immunostaining with anti-PGP9.5 antibody (\#78630504; AbD Serotec, UK) and viewed by light microscopy. Nerve profiles present in the epidermis were quantified relative to length of dermal/epidermal border examined.

Analysis. All measurements were made on coded animals by observers who were unaware of the study group or treatment regimen. Data are shown as group mean \pm S.D. Statistical comparison of experimental groups against the control + vehicle group and against the STZ + vehicle group at a single, predefined time point was made by one-way ANOVA followed by Sidak's post hoc test. Statistical comparisons of groups across time were made by two-way repeated measures ANOVA with between-group differences identified by Dunnett's post hoc test.

\section{Results}

Adult female $\mathrm{C} 57 \mathrm{Bl} / 6 \mathrm{~J}$ mice received a single dose of pirenzepine dihydrochloride (Sigma) by subcutaneous injection (10 $\mathrm{mg} / \mathrm{kg})$, oral gavage $(10 \mathrm{mg} / \mathrm{kg})$, or via topical application to the right hind paw ( $50 \mu \mathrm{l}$ of $2 \%$ in hydrogel for 20 minutes). Mice were exsanguinated at $1,3,6$, or 24 hours after drug delivery and plasma was stored at $-70^{\circ} \mathrm{C}$ until assay of pirenzepine. The highest plasma concentrations were achieved following subcutaneous delivery of pirenzepine $\left(\mathrm{AUC}_{0-\mathrm{t}}=4052\right.$ hour*nM, $C_{\max }=2600 \mathrm{nM}, T_{\max }=1$ hour $)$, followed by oral administration $\left(\mathrm{AUC}_{0-\mathrm{t}}=899\right.$ hour*nM, $C_{\max }$ $=137 \mathrm{nM}, T_{\max }=1$ hour) and then topical administration $\left(\mathrm{AUC}_{0-\mathrm{t}}=136\right.$ hour*nM, $C_{\max }=15 \mathrm{nM}, T_{\max }=6$ hour: Fig. 1$)$.

To determine whether pirenzepine applied topically to the hind paw could prevent onset of indices of neuropathy in a rodent model of diabetes, adult female $\mathrm{C} 57 \mathrm{Bl} / 6 \mathrm{~J}$ mice were

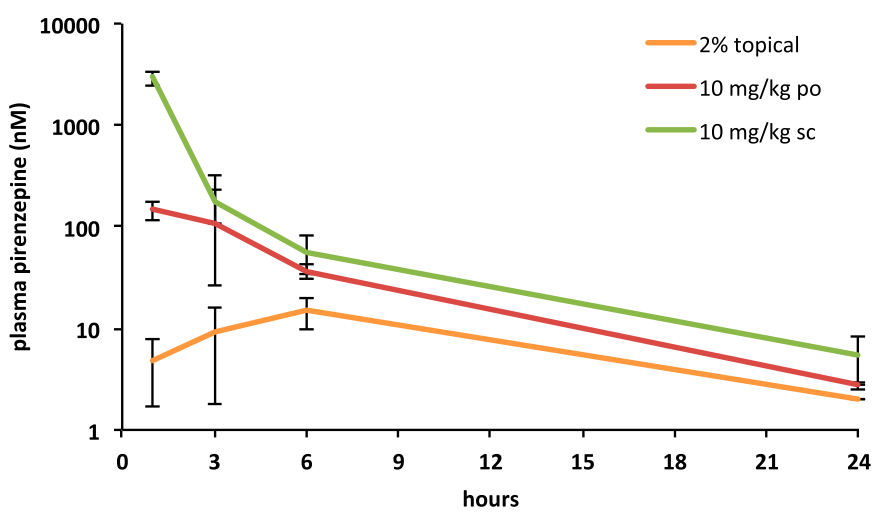

Fig. 1. Topical pirenzepine enters systemic circulation. Plasma pirenzepine concentration in mice following delivery of $10 \mathrm{mg} / \mathrm{kg}$ drug by subcutaneous injection (green line) or oral gavage (po, red line) vs. exposure of one hind paw to a $2 \%$ solution of drug in hydrogel (orange line) for 20 minutes. Data are means \pm S.D. of $N=3$ mice per delivery route and per time point. 
made diabetic with STZ, and upon confirmation of hyperglycemia, they were treated with vehicle or pirenzepine applied to the right hind paw $(0.2 \%, 1.0 \%$, or $2.0 \%$ in $50 \mu$ l hydrogel, $20 \mathrm{~min} /$ day $\times 5$ days $/ \mathrm{wk})$ for 8 weeks. The left paw was untreated. Pirenzepine treatment did not prevent weight loss or hyperglycemia in diabetic mice, although the significantly elevated blood glucose in diabetic mice treated with high doses of pirenzepine compared with vehicle-treated diabetic mice was not seen in later studies (Table 1). At study end, paw thermal hypoalgesia in STZ-diabetic mice was significantly prevented by treatment with $1.0 \%$ or $2.0 \%$ pirenzepine but not by $0.2 \%$ pirenzepine (Fig. $2 \mathrm{~A}$ ). A trend toward loss of IENF in paw skin was also prevented by $2 \%$ pirenzepine but not by the $0.2 \%$ or $1.0 \%$ dose formulations (Fig. 2B). MNCV slowing (trend to decrease, Fig. 2C) and paw tactile allodynia $(P<$ 0.01, Fig. 2D) in STZ-diabetic mice were not significantly protected at any dose of pirenzepine.

To establish the duration that efficacy persists after withdrawal of treatment and whether topical pirenzepine could reverse established neuropathy, adult female $\mathrm{C} 57 \mathrm{Bl} / 6 \mathrm{~J}$ mice were made diabetic with STZ, and upon confirmation of hyperglycemia, were treated with $2.0 \%$ pirenzepine or vehicle applied to the right hind paw, $20 \mathrm{~min} /$ day $\times 5$ days/wk. The left paw was untreated. Paw response latency to heating was selected as the index of treatment efficacy (Fig. 2A) because it is amenable to repeated testing over time. Pirenzepine treatment of diabetic mice had no effect on body weight or blood glucose levels in either control or diabetic mice (Table 1). After 8 weeks of treatment, paw heat hypoalgesia present in vehicle-treated diabetic mice was prevented in diabetic mice treated with pirenzepine (Fig. 3A). Pirenzepine treatment was then withdrawn and replaced with treatment by vehicle. Serial testing of paw heat response latency demonstrated a slowly progressing loss of heat sensation that stabilized by week 16 of diabetes (Fig. 3A). At this point, diabetic mice that had received only vehicle were transferred to topical treatment with $2.0 \%$ pirenzepine to the paw $(20 \mathrm{~min} /$ day $\times 5$ days/ wk). Paw heat hypoalgesia was completely reversed after 8 weeks of treatment (Fig. 3A). In a separate study to establish optimal dose frequency, adult female Swiss Webster mice were made diabetic with STZ and, upon confirmation of hyperglycemia, were treated with $50 \mu$ l of $2.0 \%$ pirenzepine or vehicle applied to the right hind paw, $20 \mathrm{~min} /$ day for either 1,3 , or 5 days/wk for 10 weeks. The left paw was untreated. Pirenzepine treatment of diabetic mice had no effect on body weight or hyperglycemia compared with vehicle-treated diabetic mice (Table 1). Paw heat hypoalgesia present in vehicletreated mice was prevented in a dose frequency-dependent manner, with dosing 5 days/wk completely normalizing paw heat response latency (Fig. 3B), confirming data obtained with C57 Bl/6J mice (Fig. 2A).

To determine whether higher doses of pirenzepine impacted indices of neuropathy not responsive to $2.0 \%$ topical pirenzepine (Fig. 2) and were capable of having systemic effects, adult female STZ-diabetic C57 Bl/6J mice were treated from onset of hyperglycemia with vehicle or pirenzepine applied to the right hind paw $(2.0 \%$ or $10.0 \%$ in $50 \mu$ l hydrogel, $20 \mathrm{~min} /$ day $\times$ 5 days/wk for 8 weeks). The left paw was treated with vehicle. Pirenzepine treatment of diabetic mice had no effect on body weight or hyperglycemia compared with vehicle-treated diabetic mice (Table 1). At study end, vehicle-treated diabetic mice showed bilateral MNCV slowing (Fig. 4A), paw allodynia to von Frey filaments (Fig. 4B), paw thermal hypoalgesia (Fig. 4C), and loss of sensory nerves in paw skin (Fig. 4D). Topical pirenzepine $(2.0 \%)$ prevented paw tactile allodynia, heat hypoalgesia, and loss of IENF in the treated paw but had
A
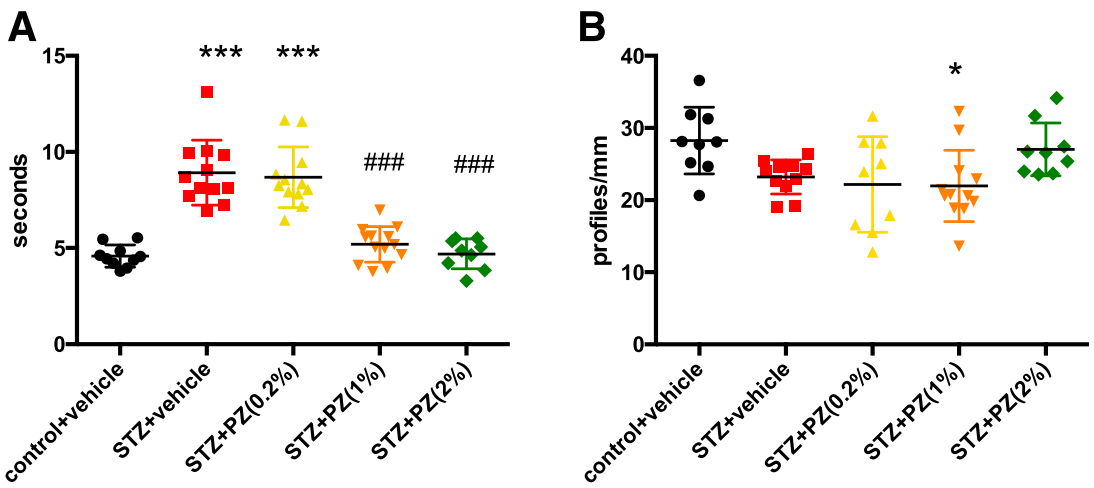

C

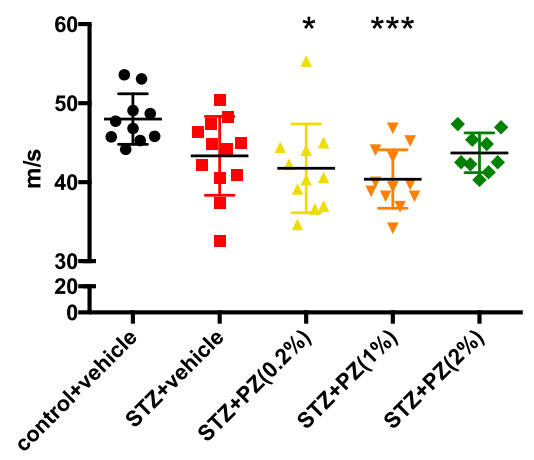

D

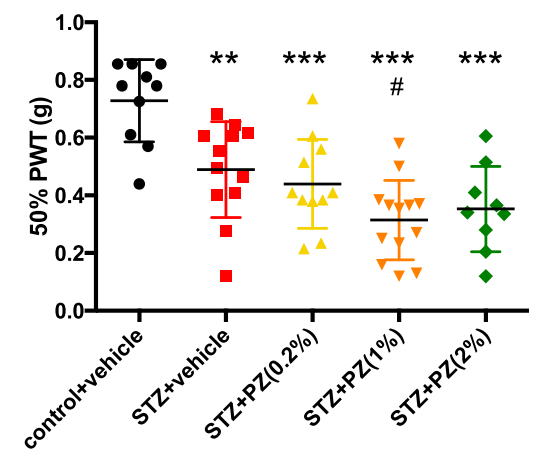

Fig. 2. Topical pirenzepine (PZ) dose-dependently prevents small fiber neuropathy in diabetic mice. Paw heat response latency (A), IENF profile density (B), motor nerve conduction velocity (C), and paw response to von Frey filaments (D) in control and STZ-diabetic mice treated with vehicle or $0.2 \%-2.0 \%$ pirenzepine in hydrogel applied to the hind paw for $20 \mathrm{~min} /$ day, 5 days/ wk, for 12 weeks. Data are group means \pm S.D. of $N=9$-13/group. $* P<0.05$; $* * P<0.01 ; * * * P<0.001$ vs.

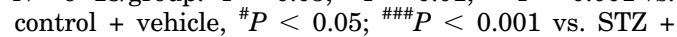
vehicle by one-way ANOVA with Sidak's post hoc test. 

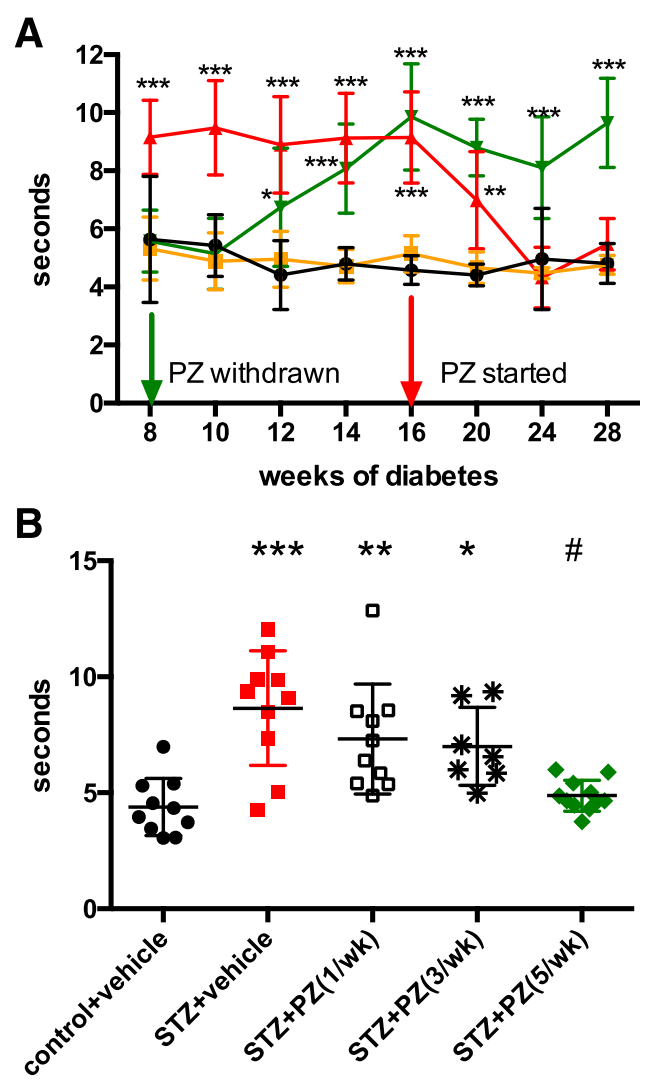

Fig. 3. Effects of topical pirenzepine dose regimen on paw heat hypoalgesia. (A) Paw heat response latency in control + vehicle (black line), control $+2.0 \%$ pirenzepine (gold line), and STZ-diabetic mice \pm $2.0 \%$ pirenzepine (PZ), either applied topically to the hind paw for $20 \mathrm{~min} /$ day, 5 days/wk, for 8 weeks from onset of hyperglycemia before stopping treatment (withdrawal group, green line) or beginning at week 16 of diabetes for a further 12 weeks (reversal group, red line). Data are group means \pm S.D. of $N=4-8$ /group. ${ }^{* *} P<0.01$; $* * * P<0.001$ vs. control + vehicle by two-way repeated measures ANOVA with Dunnett's post hoc test. (B) Paw heat response latency in control mice and STZ-diabetic mice $\pm 2.0 \%$ pirenzepine applied topically to the hind paw for $20 \mathrm{~min} / \mathrm{day}, 1,3$, or 5 days/wk, for 10 weeks from onset of hyperglycemia. Data are group means \pm S.D. of $N=7-11$ /group. ${ }^{*} P<0.05 ; * * P<0.01 ; * * * P<0.001$ vs. control + vehicle, ${ }^{\#} P<0.05$ vs. STZ + vehicle by one-way ANOVA with Sidak's post hoc test.

no impact on MNCV slowing in the ipsilateral limb. Similar efficacy was noted in the vehicle-treated contralateral limb. Increasing pirenzepine dose to $10.0 \%$ prevented all measured indices of neuropathy, including MNCV slowing, in both treated and untreated contralateral limbs.

To determine whether efficacy of pirenzepine extended to another antimuscarinic agent and whether local and systemic efficacy could be achieved when delivery was topical to the eye, adult female Swiss Webster mice were treated from onset of hyperglycemia with atropine applied to either the right hind paw $(2.0 \%$ in $50 \mu l$ hydrogel, $20 \mathrm{~min} /$ day $\times 5$ days/wk for 8 weeks) or the right eye (2.0\% in ophthalmic gel $\times 5$ days/wk for 8 weeks). Vehicle was applied to the contralateral eye or paw and also to groups of control and STZ-diabetic mice. Atropine treatment of diabetic mice by either route had no effect on body weight or hyperglycemia compared with vehicle-treated diabetic mice (Table 1). Diabetes induced significant MNCV slowing (Fig. 5A), tactile allodynia (Fig. 5B), heat hypoalgesia (Fig. 5C), significantly reduced IENF profile density (Fig. 5D), and corneal sub-basal nerve plexus density (Fig. 5E). Atropine delivered to the paw prevented MNCV slowing, heat hypoalgesia, and loss of IENF in the ipsilateral limb, but not in the contralateral, vehicle-treated limb, and had no effect on paw tactile allodynia or corneal nerve density in either limb or eye. Atropine delivered to the eye prevented loss of corneal subbasal nerve plexus density in the treated eye but not the contralateral, vehicle-treated eye. Ocular delivery of atropine also significantly prevented or attenuated paw heat hypoalgesia in both hind paws, but it did not prevent MNCV slowing, tactile allodynia, or loss of IENF.

\section{Discussion}

Clinical use of muscarinic antagonists is associated with side effects due to the widespread distribution of MR subtypes across organ systems, lack of receptor subtype specificity of antagonists, and in some cases, production of toxic metabolites. Strategies to mitigate side effects of antimuscarinics include development of receptor subtype specific (or highly selective) compounds that have limited distribution and tissue penetration profiles. For example, oral delivery of the hydrophilic $\mathrm{M}_{1} \mathrm{R}$-selective antagonist pirenzepine was developed to allow local delivery to the alimentary tract for treatment of ulcers (Carmine and Brogden, 1985; Stockbrugger, 1988). Similarly, the development history of oxybutynin to treat overactive bladder shows progression from oral to topical transdermal gel and patch formulations to avoid first pass metabolism in the upper gastrointestinal tract and liver, thereby reducing production of metabolites associated with side effects while maintaining systemic therapeutic levels of the parent compound (Sand, 2009). Our discovery studies that demonstrated the therapeutic potential of muscarinic antagonists in rodent models of peripheral neuropathy focused on subcutaneous delivery (Calcutt et al., 2017) to obtain comprehensive systemic exposure of pirenzepine, a $M_{1} R$-selective muscarinic antagonist with low central nervous system penetrance (Jaup and Blomstrand, 1980; Sethy and Francis, 1990). We have extended these discovery studies by investigating the efficacy of topically delivered pirenzepine against multiple indices of neuropathy in a mouse model of diabetes. There is precedence for the clinical viability of this approach, as topical delivery of antimuscarinics is currently used to treat overactive bladder (oxybutynin) and hyperhidrosis (glycopyrrolate).

Topical delivery of pirenzepine to the paw dose-dependently prevented multiple indices of peripheral neuropathy in the ipsilateral limb of diabetic mice. Assays were performed 24 hours after the last delivery of pirenzepine, a time when drug has cleared from circulation, suggesting long-acting effects. This is consistent with recent studies demonstrating that $\mathrm{M}_{1} \mathrm{R}$ antagonists activate an AMPK-dependent energy sensing signaling cascade driving mitochondrial function in sensory neurons and modulating microtubule polymerization in these cells (Calcutt et al., 2017; Sabbir et al., 2018; Sabbir and Fernyhough, 2018). There were some notable differences in the dose required to protect against specific indices of diabetic neuropathy. For example, paw heat hypoalgesia was prevented at topical pirenzepine doses of $1.0 \%$ or higher, whereas preventing loss of the epidermal small sensory fibers that transduce heat pain sensation required $2.0 \%$ pirenzepine or higher. This dissociates impact of pirenzepine on nerve density and function at the $1.0 \%$ dose and suggests that factors 
A
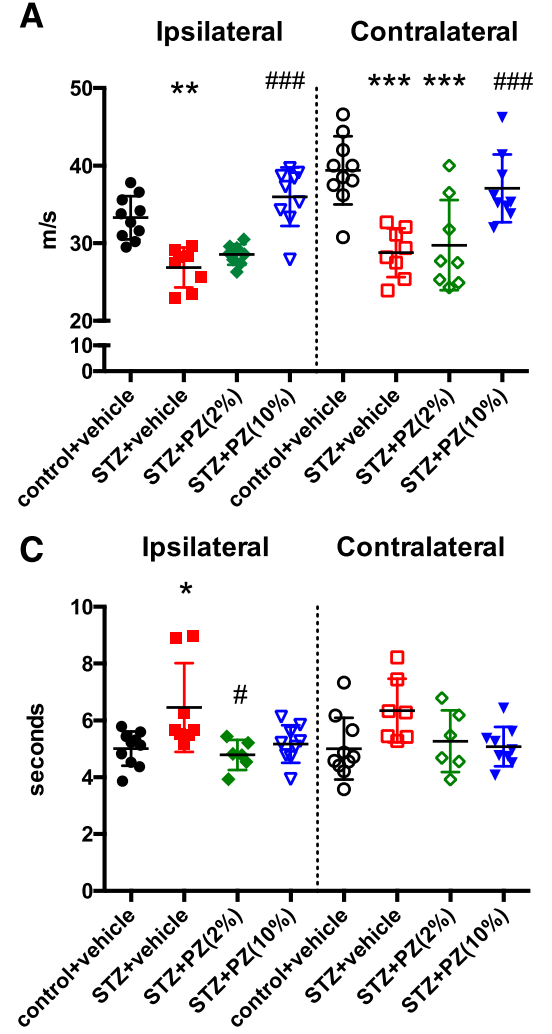

B
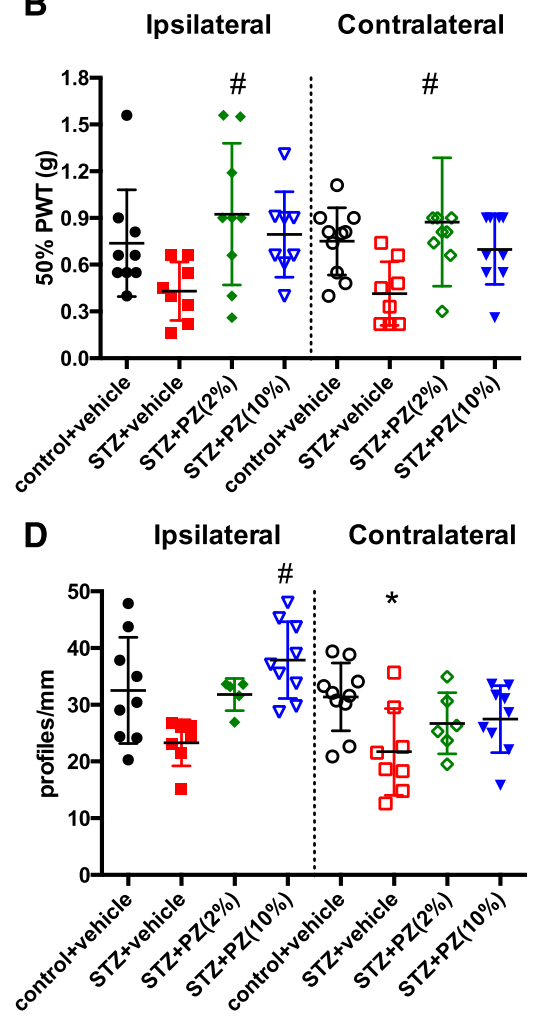

Fig. 4. Systemic effects of topical pirenzepine (PZ). STZ-diabetic mice were treated from onset of diabetes with vehicle or $2.0 \%-10.0 \%$ pirenzepine in hydrogel applied to the hind paw for $20 \mathrm{~min} /$ day, 5 days/wk. Motor nerve conduction velocity (A) and paw response to von Frey filaments (PWT: Paw withdrawal threshold) (B) were measured after 4 weeks of treatment, and paw response to heat (C) and IENF profile density (D) were measured after 8 weeks of treatment. Measurements were made in both the treated paw and the contralateral vehicle-treated paw. Data are group means \pm S.D. of $N=7-10 /$ group. $* P<0.05 ; * * P<0.01 ; * * * P<0.001 \mathrm{vs}$

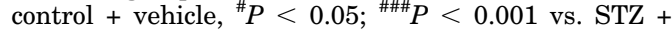
vehicle by one-way ANOVA with Sidak's post hoc test. other than global IENF depletion contribute to loss of heat sensation in diabetic mice. There is precedence for this disassociation, as paw thermal hypoalgesia occurs in shortterm diabetic mice in the absence of concurrent IENF loss (Beiswenger et al., 2008; Jolivalt et al., 2015). Neurochemical consequences of diabetes, such as impaired synthesis, axonal transport, and release of neuropeptides associated with thermal pain transmission (Diemel et al., 1994; Fernyhough et al., 1994; Calcutt et al., 1998, 2000), may contribute to thermal hypoalgesia prior to detectable IENF depletion. It is also possible that there is impact of pirenzepine on select subtypes of IENF that is not discriminated when all IENFs are quantified using the pan-neuronal marker PGP9.5 (Beiswenger et al., 2008).

Diabetic neuropathy is frequently described as a small fiber neuropathy because of the early appearance of positive and negative symptoms of small fiber dysfunction (Oaklander and Nolano, 2019). However, indices of large myelinated fiber dysfunction, such as sensory and motor nerve conduction slowing, are also early features of human diabetic neuropathy (Bril, 2016), albeit of less immediate concern to the patient, although large fiber demyelination and axonal degeneration and loss are established pathologic features (Malik, 2014). Topical pirenzepine therapy exhibited a dose-dependent capacity to prevent allodynia to von Frey filaments in STZdiabetic mice, with no effect of doses at or below $1.0 \%$ and efficacy in both treated and untreated contralateral limbs at higher doses. The intermediate dose of $2.0 \%$ showed efficacy in only one of two replicate experiments, a finding for which we currently have no explanation. As allodynia persists in C-fiber-deficient diabetic rodents (Khan et al., 2002), this disorder likely involves large sensory fibers. We have previously demonstrated that prevention of allodynia in STZ-diabetic rats by pirenzepine is not an acute phenomenon (Calcutt et al., 2017). The concept of gradual regulation of neuronal phenotype is also supported in small sensory fibers in which reversal of paw thermal hypoalgesia took 4-8 weeks; withdrawal of pirenzepine precipitated onset of hypoalgesia over a similar time course. Our prior studies aligned neuroprotection by antimuscarinics with upregulation of AMPK signaling and preservation of mitochondrial bioenergetics (Calcutt et al., 2017). $M_{1} R$ antagonism also removes a constraint on microtubule polymerization and mitochondrial trafficking (Sabbir et al., 2018) and promotes a biased $\beta$-arrestin-dependent extracellular signal-regulated kinase-cAMP-responsive element binding protein (ERK-CREB) signal (Sabbir and Fernyhough, 2018) so that both blocking growth-impeding signals and initiating other signaling cascades may contribute to neuroprotection. Whether autonomic neuropathy (Schmidt, 2014) is afforded similar protection remains to be determined.

The neuroprotective properties of pirenzepine extended to dose-dependent preservation of large fiber MNCV, albeit requiring doses higher than those for protection of tactile allodynia and thermal hypoalgesia. It is notable that MNCV was preserved only at topical doses of $10.0 \%$ pirenzepine, whereas small fiber function (heat sensation) and structure (IENF) were normalized at doses as low as $1.0 \%-2.0 \%$. It remains to be established whether this is an intrinsic feature of the different components of the peripheral nervous system or simply reflects the relative ease of access of pirenzepine to sensory neuronal terminals in the epidermis and/or cell bodies in the dorsal root ganglia versus motor nerve terminals in muscle and/or their cell bodies in the ventral spinal cord. Our finding that topical delivery of $10.0 \%$ pirenzepine to one hind paw was neuroprotective in the contralateral paw certainly suggests that there is a systemic component to the effects of 
A
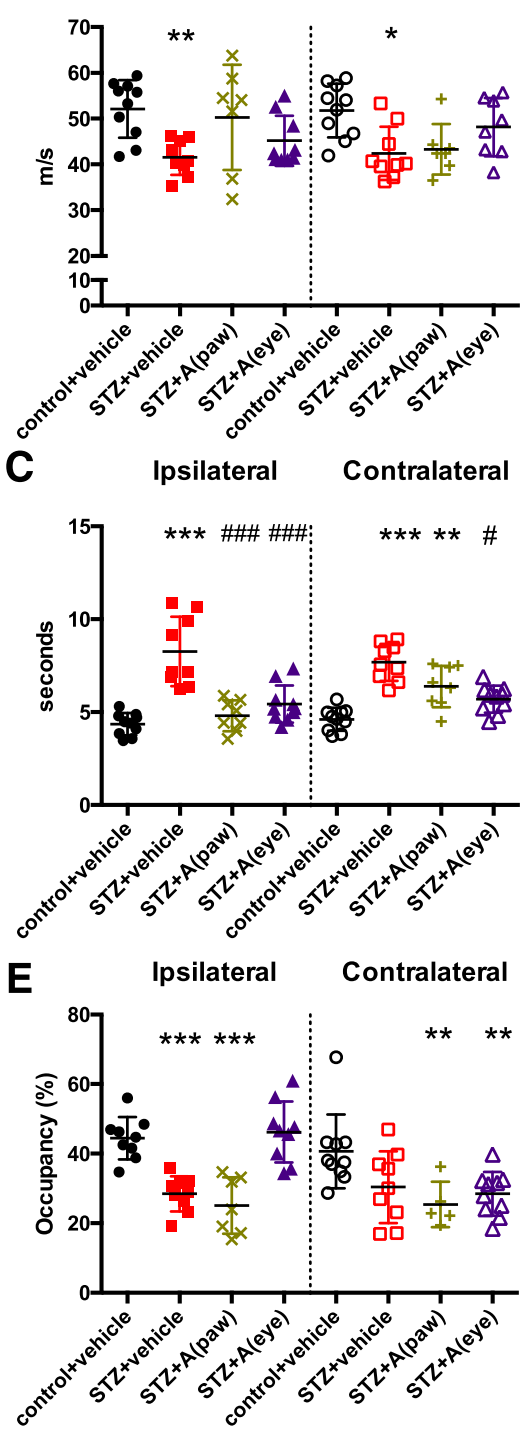

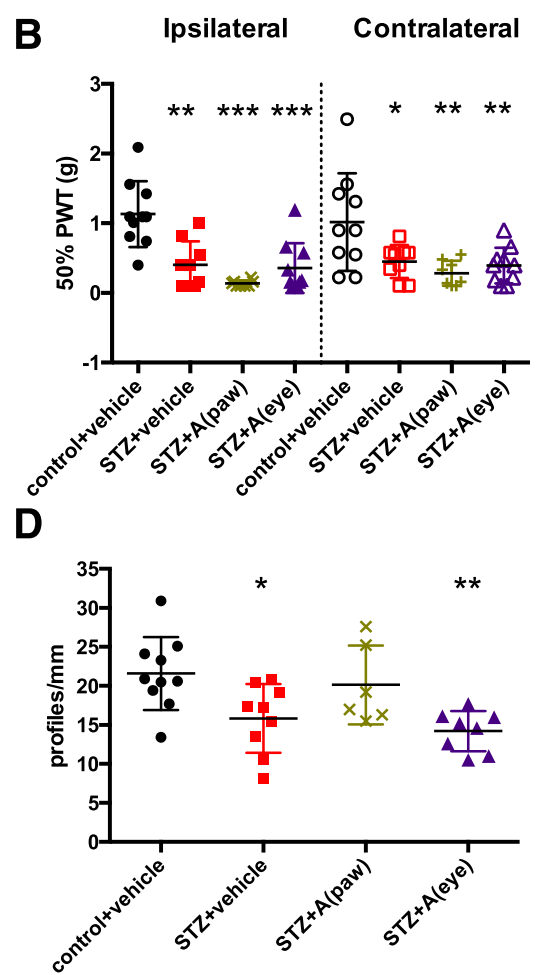

Fig. 5. Systemic effects of topical atropine (A). STZdiabetic mice were treated from onset of diabetes with vehicle or $2.0 \%$ atropine (A) in hydrogel applied to the hind paw for $20 \mathrm{~min} /$ day, 5 days/wk, or to the eye (50 $\mu \mathrm{l}$ in saline) 5 days/wk. Motor nerve conduction velocity (A), paw response to von Frey filaments (B), paw response to heat (C), IENF profile density (D), and occupancy of corneal nerves of the sub-basal nerve plexus were measured after 12 weeks of treatment. Measurements were made in both the treated paw/eye and the contralateral vehicle-treated paw/eye, except for IENF density, for which only tissue from the treated paw was collected. Data are group means \pm S.D. of $N=$ 6-10/group. $* P<0.05 ; * * P<0.01 ; * * * P<0.001$ vs. control + vehicle, ${ }^{\#} P<0.05 ;{ }^{\# \#} P<0.01 ;{ }^{\# \# \#} P<0.001$ vs. STZ + vehicle by one-way ANOVA with Sidak's post hoc test topical pirenzepine. Topical $2.0 \%$ pirenzepine had a $C_{\max }$ of 15 $\mathrm{nM}$, matching concentrations that promote increased neurite outgrowth from adult sensory neurons in vitro (Calcutt et al., 2017). This may therefore represent a target therapeutic concentration for actions on small sensory neurons, although we cannot exclude indirect actions of pirenzepine in vivo.

Although $\mathrm{M}_{1} \mathrm{R}$-specific and -selective antagonists have therapeutic potential, there are a number of less-selective muscarinic antagonists in current clinical use. Atropine antagonizes all five muscarinic receptor subtypes, and topical atropine, acting via $\mathrm{M}_{3} \mathrm{R}$, is used as a mydriatic and cycloplegic during ophthalmic procedures. Atropine delivered to the paw of diabetic mice replicated the effects of pirenzepine in that it prevented MNCV slowing, heat hypoalgesia, and loss of IENF in the ipsilateral limb. However, there was not complete concordance, as atropine was effective against MNCV slowing at a lower dose $(2.0 \%)$ than pirenzepine $(10.0 \%)$ but did not prevent paw tactile allodynia at any dose. Further, the effects of $2.0 \%$ pirenzepine on indices of neuropathy in the contralateral limb were not observed when using $2.0 \%$ atropine. The extent to which these differences reflect chemical and/or pharmacological differences in the two muscarinic antagonists requires investigation. Given the widespread ophthalmic use of atropine in humans, we also investigated the consequences of delivery to the eye on both local corneal sensory nerves and more-distant sensory and motor nerves. Systemic efficacy of drugs delivered to the eye has precedence, as topical delivery of insulin has systemic effects on glucose metabolism (Liu and Chiou, 1994). Loss of corneal nerve density in diabetic mice was completely prevented by atropine, but only in the treated eye. Delivery of atropine to the eye also prevented paw heat hypoalgesia and had minor effects on MNCV slowing, but without effect on paw IENF density. These data reinforce the disassociation of paw thermal sensation and IENF density discussed above and demonstrate that topical delivery of atropine to the eye can produce effects on systemic indices of neuropathy that are most sensitive to muscarinic antagonists. Measuring corneal nerve density has been championed as a biomarker for generalized small sensory fiber neuropathy (Petropoulos et al., 2019), and there is good evidence that corneal nerve density recovers in advance of other indices of neuropathy in patients cured of diabetes following pancreatic 
transplantation (Azmi et al., 2019). However, as topical atropine to the paw prevented multiple indices of neuropathy in the treated limb but did not prevent corneal nerve loss, caution should be exercised when considering using corneal nerve density as the sole arbiter of therapeutic efficacy against small fiber neuropathies.

The muscarinic antagonists pirenzepine and atropine exhibited broad-spectrum neuroprotective properties against multiple indices of peripheral neuropathy when applied to the paw of diabetic mice. Efficacy was notable in the treated limb but, for pirenzepine in particular, was also evident in the untreated contralateral limb. Discord in the therapeutic profiles of pirenzepine and atropine may be the result of their distinct chemical and pharmacological properties. These data offer a plausible therapeutic approach for the use of antimuscarinics to treat diabetic neuropathy. The availability of topical formulations of antimuscarinics may facilitate evaluation of the translational potential of this therapeutic approach for a condition that has no Food and Drug Administration-approved medication.

\section{Authorship Contributions}

Participated in research design: Frizzi, Kotra, Fernyhough, Calcutt.

Conducted experiments: Frizzi, Han, Mota, Guernsey.

Performed data analysis: Jolivalt, Kotra, Calcutt.

Wrote or contributed to the writing of the manuscript: Jolivalt, Fernyhough, Calcutt.

\section{References}

Azmi S, Jeziorska M, Ferdousi M, Petropoulos IN, Ponirakis G, Marshall A, Alam U, Asghar O, Atkinson A, Jones W, et al. (2019) Early nerve fibre regeneration in individuals with type 1 diabetes after simultaneous pancreas and kidney transplantation Diabetologia 62:1478-1487.

Beiswenger KK, Calcutt NA, and Mizisin AP (2008) Dissociation of thermal hypoalgesia and epidermal denervation in streptozotocin-diabetic mice. Neurosci Let 442:267-272.

Bril V (2016) The perfect clinical trial. Int Rev Neurobiol 127:27-41.

Calcutt NA, Chen P, and Hua XY (1998) Effects of diabetes on tissue content and evoked release of calcitonin gene-related peptide-like immunoreactivity from rat sensory nerves. Neurosci Lett 254:129-132.

Calcutt NA, Smith DR, Frizzi K, Sabbir MG, Chowdhury SK, Mixcoatl-Zecuatl T, Saleh A, Muttalib N, Van der Ploeg R, Ochoa J, et al. (2017) Selective antagonism of muscarinic receptors is neuroprotective in peripheral neuropathy. J Clin Invest 127: $608-622$

Calcutt NA, Stiller C, Gustafsson H, and Malmberg AB (2000) Elevated substance-Plike immunoreactivity levels in spinal dialysates during the formalin test in normal and diabetic rats. Brain Res 856:20-27.

Carmine AA and Brogden RN (1985) Pirenzepine. A review of its pharmacodynamic and pharmacokinetic properties and therapeutic efficacy in peptic ulcer disease and other allied diseases. Drugs 30:85-126.

Chandrasekaran K, Anjaneyulu M, Choi J, Kumar P, Salimian M, Ho CY, and Russell JW (2019) Role of mitochondria in diabetic peripheral neuropathy: influencing the $\mathrm{NAD}^{+}$-dependent SIRT1-PGC-1 $\alpha$-TFAM pathway. Int Rev Neurobiol 145:177-209.

Chaplan SR, Bach FW, Pogrel JW, Chung JM, and Yaksh TL (1994) Quantitative assessment of tactile allodynia in the rat paw. $J$ Neurosci Methods 53:55-63.

Chen DK, Frizzi KE, Guernsey LS, Ladt K, Mizisin AP, and Calcutt NA (2013) Repeated monitoring of corneal nerves by confocal microscopy as an index of peripheral neuropathy in type-1 diabetic rodents and the effects of topical insulin. J Peripher Nerv Syst 18:306-315.

Davidson E, Coppey L, Lu B, Arballo V, Calcutt NA, Gerard C, and Yorek M (2009) The roles of streptozotocin neurotoxicity and neutral endopeptidase in murine experimental diabetic neuropathy. Exp Diabetes Res 2009:431980.

Diemel LT, Brewster WJ, Fernyhough P, and Tomlinson DR (1994) Expression of neuropeptides in experimental diabetes; effects of treatment with nerve growth factor or brain-derived neurotrophic factor. Brain Res Mol Brain Res 21:171-175.

Eglen RM, Choppin A, and Watson N (2001) Therapeutic opportunities from muscarinic receptor research. Trends Pharmacol Sci 22:409-414.

Erskine D, Taylor JP, Bakker G, Brown AJH, Tasker T, and Nathan PJ (2019) Cholinergic muscarinic $\mathrm{M}_{1}$ and $\mathrm{M}_{4}$ receptors as therapeutic targets for cognitive, behavioural, and psychological symptoms in psychiatric and neurological disorders. Drug Discov Today 24:2307-2314.

Fernyhough P (2015) Mitochondrial dysfunction in diabetic neuropathy: a series of unfortunate metabolic events. Curr Diab Rep 15:89.

Fernyhough P, Diemel LT, Brewster WJ, and Tomlinson DR (1994) Deficits in sciatic nerve neuropeptide content coincide with a reduction in target tissue nerve growth factor messenger RNA in streptozotocin-diabetic rats: effects of insulin treatment. Neuroscience 62:337-344.
Gautam D, Han SJ, Heard TS, Cui Y, Miller G, Bloodworth L, and Wess J (2005) Cholinergic stimulation of amylase secretion from pancreatic acinar cells studied with muscarinic acetylcholine receptor mutant mice. $J$ Pharmacol Exp Ther 313: 995-1002.

Giglio D and Tobin G (2009) Muscarinic receptor subtypes in the lower urinary tract. Pharmacology 83:259-269.

Harrington AM, Peck CJ, Liu L, Burcher E, Hutson JM, and Southwell BR (2010) Localization of muscarinic receptors M1R, M2R and M3R in the human colon. Neurogastroenterol Motil 22:999-1008, e262-3.

Jaup BH and Blomstrand C (1980) Cerebro-spinal fluid concentrations of pirenzepine after therapeutic dosage. Scand J Gastroenterol Suppl 66:35-37.

Jolivalt CG, Frizzi KE, Guernsey L, Marquez A, Ochoa J, Rodriguez M, and Calcutt NA (2016) Peripheral neuropathy in mouse models of diabetes. Curr Protoc Mouse Biol 6:223-255.

Jolivalt CG, Rodriguez M, Wahren J, and Calcutt NA (2015) Efficacy of a long-acting C-peptide analogue against peripheral neuropathy in streptozotocin-diabetic mice. Diabetes Obes Metab 17:781-788.

Jositsch G, Papadakis T, Haberberger RV, Wolff M, Wess J, and Kummer W (2009) Suitability of muscarinic acetylcholine receptor antibodies for immunohistochemistry evaluated on tissue sections of receptor gene-deficient mice. Naunyn Schmiedebergs Arch Pharmacol 379:389-395.

Khan GM, Chen SR, and Pan HL (2002) Role of primary afferent nerves in allodynia caused by diabetic neuropathy in rats. Neuroscience 114:291-299.

Krajewski JL, Dickerson IM, and Potter LT (2001) Site-directed mutagenesis of m1toxin1: two amino acids responsible for stable toxin binding to $\mathrm{M}(1)$ muscarinic receptors. Mol Pharmacol 60:725-731.

Kruse AC, Kobilka BK, Gautam D, Sexton PM, Christopoulos A, and Wess J (2014) Muscarinic acetylcholine receptors: novel opportunities for drug development. Nat Rev Drug Discov 13:549-560.

Kurzen H, Berger H, Jäger C, Hartschuh W, Näher H, Gratchev A, Goerdt S, and Deichmann M (2004) Phenotypical and molecular profiling of the extraneuronal cholinergic system of the skin. J Invest Dermatol 123:937-949.

Liu SX and Chiou GC (1994) Feasibility of insulin eyedrops for human use. J Ocul Pharmacol 10:587-590.

Loreti S, Vilaró MT, Visentin S, Rees H, Levey AI, and Tata AM (2006) Rat Schwann cells express M1-M4 muscarinic receptor subtypes. J Neurosci Res 84:97-105.

Malik RA (2014) Pathology of human diabetic neuropathy. Handb Clin Neurol 126: 249-259.

Moran SP, Maksymetz J, and Conn PJ (2019) Targeting muscarinic acetylcholine receptors for the treatment of psychiatric and neurological disorders. Trends Pharmacol Sci 40:1006-1020.

Ndoye A, Buchli R, Greenberg B, Nguyen VT, Zia S, Rodriguez JG, Webber RJ, Lawry MA, and Grando SA (1998) Identification and mapping of keratinocyte muscarinic acetylcholine receptor subtypes in human epidermis. J Invest Dermatol 111: $410-416$.

Oaklander AL and Nolano M (2019) Scientific advances in and clinical approaches to small-fiber polyneuropathy: a review. JAMA Neurol DOI: 10.1001/jamaneurol.2019.2917 [published ahead of print].

Petropoulos IN, Ponirakis G, Khan A, Gad H, Almuhannadi H, Brines M, Cerami A, and Malik RA (2019) Corneal confocal microscopy: ready for prime time. Clin Exp Optom 103:265-277.

Roda RH and Hoke A (2019) Mitochondrial dysfunction in HIV-induced peripheral neuropathy. Int Rev Neurobiol 145:67-82.

Sabbir MG, Calcutt NA, and Fernyhough P (2018) Muscarinic acetylcholine type 1 receptor activity constrains neurite outgrowth by inhibiting microtubule polymerization and mitochondrial trafficking in adult sensory neurons. Front Neurosci 12: 402

Sabbir MG and Fernyhough P (2018) Muscarinic receptor antagonists activate ERKCREB signaling to augment neurite outgrowth of adult sensory neurons. Neuropharmacology 143:268-281.

Sand PK (2009) The evolution of transdermal therapy for overactive bladder. Curr Urol Rep 10:338-341.

Saternos HC, Almarghalani DA, Gibson HM, Meqdad MA, Antypas RB, Lingireddy A, and AbouAlaiwi WA (2018) Distribution and function of the muscarinic receptor subtypes in the cardiovascular system. Physiol Genomics 50:1-9.

Schmidt RE (2014) Autonomic neuropathy in experimental models of diabetes mellitus. Handb Clin Neurol 126:579-602.

Servent D, Blanchet G, Mourier G, Marquer C, Marcon E, and Fruchart-Gaillard C (2011) Muscarinic toxins. Toxicon 58:455-463.

Sethy VH and Francis JW (1990) Pharmacokinetics of muscarinic cholinergic drugs as determined by ex vivo (3H)-oxotremorine-M binding. J Pharmacol Methods 23: 285-296.

Stockbrügger RW (1988) Clinical significance of M1 receptor antagonists. Pharmacology 37 (Suppl 1):54-63.

Tata AM, Tripiciano A, Filippini A, Biagioni S, and Augusti-Tocco G (2000a) Muscarinic receptors modulate intracellular calcium level in chick sensory neurons. Brain Res 866:65-72.

Tata AM, Vilaró MT, and Mengod G (2000b) Muscarinic receptor subtypes expression in rat and chick dorsal root ganglia. Brain Res Mol Brain Res 82:1-10.

Tobin G, Giglio D, and Lundgren O (2009) Muscarinic receptor subtypes in the alimentary tract. J Physiol Pharmacol 60:3-21.

Trecarichi A and Flatters SJL (2019) Mitochondrial dysfunction in the pathogenesis of chemotherapy-induced peripheral neuropathy. Int Rev Neurobiol 145:83-126.

Address correspondence to: Dr. Corinne G. Jolivalt, Department of Pathology, University of California San Diego, 9500 Gilman Dr., La Jolla, CA 92093. E-mail: cjolivalt@ucsd.edu 